\title{
Notes on homogeneous vector bundles over complex flag manifolds
}

\author{
Sergei Igonin
}

\begin{abstract}
Let $P$ be a parabolic subgroup of a semisimple complex Lie group $G$ defined by a subset $\Sigma$ of simple roots of $G$, and let $\mathbf{E}_{\varphi}$ be a homogeneous vector bundle over the flag manifold $G / P$ corresponding to a linear representation $\varphi$ of $P$. Using Bott's theorem, we obtain sufficient conditions on $\varphi$ in terms of the combinatorial structure of $\Sigma$ for some cohomology groups of the sheaf of holomorphic sections of $\mathbf{E}_{\varphi}$ to be zero. In particular, we define two numbers $d(P), \ell(P) \in \mathbb{N}$ such that for any $\varphi$ obtained by natural operations from a representation of dimension less than $d(P)$ the $q$-th cohomology group of $\mathbf{E}_{\varphi}$ is zero for $0<q<\ell(P)$. We prove also that in this case that the vector bundle $\mathbf{E}_{\varphi}$ is rigid.
\end{abstract}

Let $\mathbf{E}$ be a holomorphic vector bundle over a connected compact complex manifold $M$. Then there is a natural homomorphism of complex Lie groups $\mu:$ Aut $\mathbf{E} \rightarrow$ $\operatorname{Bih} M$, where Aut $\mathbf{E}$ is the automorphism group of the vector bundle and $\operatorname{Bih} M$ is the group of all biholomorphic transformations of $M$. The bundle $\mathbf{E}$ is said to be homogeneous if the action $\mu$ of Aut $\mathbf{E}$ on $M$ is transitive.

Assume that we have a homomorphism $\Phi: G \rightarrow$ Aut $\mathbf{E}$ such that the action $\mu \Phi$ of $G$ on $M$ is transitive. Then $\mathbf{E}$ is homogeneous; we say that $\mathbf{E}$ is homogeneous with respect to $G$. Let $o \in M$ and let $P=G_{o}$ be the stabilizer of $o$ in $G$. Then $P$ acts in a natural way on the fibre $E=E_{o}$, that is, we have a holomorphic linear representation $\varphi: P \rightarrow \mathrm{GL}(E)$. It is known that the bundle $\mathbf{E}$ is uniquely determined by the group $G$, the subgroup $P$, and the representation $\varphi$, which can all be arbitrary. We denote by $\mathbf{E}_{\varphi}$ the homogeneous vector bundle over $M=G / P$ defined by a representation $\varphi$ of $P$.

Note also that corresponding to standard tensor operations on representations there are similar operations on vector bundles with a fixed base. In particular,

$$
\mathbf{E}_{\varphi^{*}}=\mathbf{E}_{\varphi}^{*}, \quad \mathbf{E}_{\varphi_{1}+\varphi_{2}}=\mathbf{E}_{\varphi_{1}} \oplus \mathbf{E}_{\varphi_{2}}, \quad \mathbf{E}_{\varphi_{1} \varphi_{2}}=\mathbf{E}_{\varphi_{1}} \otimes \mathbf{E}_{\varphi_{2}} .
$$

For a Lie group $G$ denote by $G^{\circ}$ the identity component of $G$. We consider the case when $M$ is a flag manifold, that is, when $M$ is homogeneous and the stabilizers $(\operatorname{Bih} M)_{x}^{\circ} \subset(\operatorname{Bih} M)^{\circ}, x \in M$, are parabolic subgroups (see [1, 6]). Then the group Bih $M$ and all its transitive subgroups are semisimple Lie groups.

1991 Mathematics Subject Classification. Primary 32M10, 32L10, 17B10, 17B20.

Key words and phrases. Homogeneous vector bundle, complex flag manifold, cohomology of the sheaf of sections, Bott's theorem, root system, Dynkin diagram, rigid vector bundle.

Partially supported by the RFBR grants 01-01-00709, 00-01-00705. 
Let $\mathbf{E} \rightarrow M$ be a homogeneous vector bundle and let $Q=\mu\left((\text { Aut } \mathbf{E})^{\circ}\right)$. Then $Q$ acts on $M$ transitively and, therefore, is semisimple. By Levi's theorem, there exists a connected Lie subgroup $G$ of Aut $\mathbf{E}$ such that $\mu: G \rightarrow Q$ is a local isomorphism. Thus $\mathbf{E}$ is homogeneous with respect to a connected semisimple Lie group $G$ whose action on $M$ is locally effective.

We choose a maximal torus $T$ of $G$ and denote by $\mathfrak{t}$ the corresponding Cartan subalgebra of the tangent algebra $\mathfrak{g}$ of $G$. Let $\Delta \subset \mathfrak{t}^{*}$ be the root system associated with $T$ and let $\Delta_{+} \subset \Delta$ be a subset of positive roots. We denote by $\Pi$ the corresponding system of simple roots.

As usually, consider a non-degenerate $G$-invariant scalar product in $\mathfrak{g}$ inducing a non-degenerate scalar product $(\cdot, \cdot)$ in $\mathfrak{t}^{*}$ invariant under the Weyl group $W$. An element $\beta \in \mathfrak{t}^{*}$ is called a weight if

$$
\frac{2(\beta, \alpha)}{(\alpha, \alpha)} \in \mathbb{Z} \quad \text { for all } \alpha \in \Delta .
$$

A weight $\beta$ is said to be dominant if $(\beta, \alpha) \geq 0$ for each $\alpha \in \Delta^{+}$.

Let $\mathfrak{g}_{\alpha}$ be the root subspace of $\mathfrak{g}$ corresponding to $\alpha \in \Delta$. If $R$ is a Lie subgroup of $G$ normalized by $T$, then its tangent subalgebra $\mathfrak{r} \subset \mathfrak{g}$ has the following form:

$$
\mathfrak{r}=(\mathfrak{r} \cap \mathfrak{t}) \oplus \bigoplus_{\alpha \in \Delta(R)} \mathfrak{g}_{\alpha},
$$

where $\Delta(R) \subset \Delta$ is some subset called the root system of $R$. In particular, each system of positive roots $\Delta_{+}$determines two Borel (that is, maximal solvable) subgroups $B_{ \pm}$of $G$ containing $T$ with root systems $\Delta\left(B_{ \pm}\right)= \pm \Delta_{+}$.

Let $P$ be a parabolic subgroup of $G$, that is, a subgroup containing a Borel subgroup. We assume that $P$ contains the subgroup $B_{-}$corresponding to the system of negative roots $\Delta_{-}=-\Delta_{+}$. It is known (see [1, 6]) that $\Delta(P)=\Delta_{-} \cup[\Sigma]$ in this case, where $[\Sigma]$ is the set of all roots of $G$ that can be expressed as a linear combination of elements of a subset $\Sigma \subset \Pi$. We have the semidirect decomposition

$$
P=H \ltimes N_{-}, \quad \mathfrak{p}=\mathfrak{h} \boxplus \mathfrak{n}_{-},
$$

where $H$ is a maximal reductive subgroup, $N_{-}$is the unipotent radical of $P$, and $\mathfrak{p}, \mathfrak{h}, \mathfrak{n}_{-}$are the corresponding Lie algebras. Moreover,

$$
\begin{aligned}
\Delta(H) & =[\Sigma], \\
\Delta\left(N_{-}\right) & =\Delta_{-} \backslash[\Sigma],
\end{aligned}
$$

and $\Sigma$ coincides with the system of simple roots of the group $H$ corresponding to its Borel subgroup $B_{+} \cap H$. Thus the parabolic subgroup $P \subset G$ is uniquely determined by the subset $\Sigma \subset \Pi$ of simple roots.

Note that $T$ is also a maximal torus of $P$. Each weight of a representation $\varphi$ of $P$ is a weight in the above sense. A representation $\varphi$ is completely reducible if and only if it is trivial on $N_{-}$; in this case it is uniquely determined by the representation $\left.\varphi\right|_{H}$ of $H$. A highest weight of $\varphi$ is, by definition, a highest weight of $\left.\varphi\right|_{H}$ understood in the sense of the ordering corresponding to the Borel subgroup $B_{+} \cap H$.

One uses Bott's well-known theorem (see [1, 2]) to calculate the graded cohomology space $H^{*}\left(M, \mathcal{E}_{\varphi}\right)$, where $M=G / P$ is a flag manifold and $\mathcal{E}_{\varphi}$ is the sheaf of holomorphic sections of the homogeneous vector bundle $\mathbf{E}_{\varphi} \rightarrow M$ defined by a representation $\varphi: P \rightarrow \mathrm{GL}(E)$. 
A weight $\lambda \in \mathfrak{t}^{*}$ is said to be singular if there exists a root $\alpha \in \Delta_{+}$such that $(\lambda, \alpha)=0$, and it is said to be regular otherwise. We set $\gamma=\frac{1}{2} \sum_{\alpha \in \Delta_{+}} \alpha$; then

$$
(\gamma, \alpha)=\frac{1}{2}(\alpha, \alpha) \text { for each } \alpha \in \Pi .
$$

If $\lambda$ is a regular weight, then there exists a unique $w \in W$ such that $w(\lambda)-\gamma$ is dominant. The index of the regular weight $\lambda$ is the smallest integer $s$ such that $w$ expands into the product of $s$ reflections $s_{\alpha}, \alpha \in \Pi$. This is equal to the number of $\alpha \in \Delta_{+}$such that $(\lambda, \alpha)<0$. To each weight $\lambda \in \mathfrak{t}^{*}$ such that $\lambda+\gamma$ is regular we assign a dominant weight $I(\lambda) \in \mathfrak{t}^{*}$ by the rule $I(\lambda)=w(\lambda+\gamma)-\gamma$.

To formulate Bott's theorem, note that in each cohomology space $H^{q}(M, \mathcal{E})$ associated with a homogeneous vector bundle $\mathbf{E} \rightarrow M=G / P$ there exists a natural structure of a $G$-module.

BotT's THEOREM. Let $\varphi$ be an irreducible finite-dimensional representation of $P$ with highest weight $\Lambda$. Then the graded cohomology space $H^{*}\left(M, \mathcal{E}_{\varphi}\right)$ is determined by $\Lambda$ in the following way:

- if $\Lambda+\gamma$ is singular then $H^{*}\left(M, \mathcal{E}_{\varphi}\right)=0$;

- if $\Lambda+\gamma$ is regular and its index is $p$, then $H^{q}\left(M, \mathcal{E}_{\varphi}\right)=0$ for all $q \neq p$, while $H^{p}\left(M, \mathcal{E}_{\varphi}\right)$ is an irreducible $G$-module with highest weight $I(\Lambda)$.

Using this theorem, we will obtain some sufficient conditions on representations $\varphi$ of $P$ for a cohomology group $H^{q}\left(M, \mathcal{E}_{\varphi}\right)$ to be zero.

Bott's theorem is not applicable directly when the representation is not completely reducible. But due to the following lemma to prove that cohomology is zero it is enough to consider completely reducible representations. For an arbitrary holomorphic representation $\varphi: P \rightarrow \mathrm{GL}(E)$ we construct a completely reducible representation $\varphi^{s}$ as follows. There is a filtration (Jordan-Hölder tower)

$$
0=E_{0} \subset E_{1} \subset \ldots \subset E_{m}=E
$$

of $E$ by invariant subspaces of $\varphi(P)$ such that for all $i=1, \ldots, m$ the induced representation $\bar{\varphi}_{i}$ in $E_{i} / E_{i-1}$ is irreducible. We set

$$
\varphi^{s}=\bar{\varphi}_{1}+\cdots+\bar{\varphi}_{m} .
$$

It is well-known that $\varphi^{s}$ does not depend on a Jordan-Hölder tower of $E$.

Lemma 1. If $H^{q}\left(M, \mathcal{E}_{\varphi^{s}}\right)=0$ for some $q \geq 0$ then $H^{q}\left(M, \mathcal{E}_{\varphi}\right)=0$.

Proof. From $H^{q}\left(M, \mathcal{E}_{\varphi^{s}}\right)=0$ and (11) we have

$$
H^{q}\left(M, \mathcal{E}_{\bar{\varphi}_{i}}\right)=0 \text { for all } i=1, \ldots, m \text {. }
$$

Denote by $\varphi_{i}$ the restriction of $\varphi$ onto the invariant subspace $E_{i}$ and consider the short exact sequences of sheaves

$$
0 \rightarrow \mathcal{E}_{\varphi_{i-1}} \rightarrow \mathcal{E}_{\varphi_{i}} \rightarrow \mathcal{E}_{\bar{\varphi}_{i}} \rightarrow 0,1 \leq i \leq m,
$$

corresponding to the exact sequences

$$
0 \rightarrow E_{i-1} \rightarrow E_{i} \rightarrow E_{i} / E_{i-1} \rightarrow 0
$$

of $P$-modules. Using the exact cohomology sequence, by induction on $i$ from (5) we get $H^{q}\left(M, \mathcal{E}_{\varphi_{i}}\right)=0$ for all $i=1, \ldots, m$. 
Each $\xi \in \mathfrak{t}^{*}$ is a linear combination of simple roots $\alpha \in \Pi$. We denote by $c_{\alpha}(\xi)$ the corresponding coefficients, that is, $\xi=\sum_{\alpha \in \Pi} c_{\alpha}(\xi) \cdot \alpha$, and set

$$
\mathrm{C}(\xi)=\left\{\alpha \in \Pi: c_{\alpha}(\xi) \neq 0\right\} \subset \Pi .
$$

The value $\sqrt{(\xi, \xi)}$ is called the length of $\xi$ and denoted by $|\xi|$. For $\delta^{1}, \delta^{2} \in \Delta_{+}$we write $\delta^{1} \leq \delta^{2}$ if $c_{\alpha}\left(\delta^{1}\right) \leq c_{\alpha}\left(\delta^{2}\right)$ for all $\alpha \in \Pi$. We need two lemmas on semisimple Lie algebras.

Lemma 2. If $\delta^{1}, \delta^{2} \in \Delta_{+}$and $\delta^{1} \leq \delta^{2}$ then there exists a sequence of positive roots $\delta_{0}, \delta_{1}, \ldots, \delta_{m}$ such that $\delta_{0}=\delta^{1}, \delta_{m}=\delta^{2}$, and $\delta_{i}-\delta_{i-1} \in \mathrm{C}\left(\delta^{2}-\delta^{1}\right)$ for all $i=1, \ldots, m$.

Proof. By induction on $\left|\mathrm{C}\left(\delta^{2}-\delta^{1}\right)\right|$, we must prove that if $\delta^{1} \neq \delta^{2}$ then there is $\alpha \in \mathrm{C}\left(\delta^{2}-\delta^{1}\right)$ such that

$$
\delta^{2}-\alpha \in \Delta_{+} \text {or } \delta^{1}+\alpha \in \Delta_{+} .
$$

We have $\left(\delta^{2}-\delta^{1}, \delta^{2}-\delta^{1}\right)>0$, hence $\left(\delta^{2}-\delta^{1}, \alpha\right)>0$ for some $\alpha \in \mathrm{C}\left(\delta^{2}-\delta^{1}\right)$. Then $\left(\delta^{2}, \alpha\right)>0$ or $\left(\delta^{1}, \alpha\right)<0$, which implies (6).

Lemma 3. Let $\mathfrak{g}_{1}, \ldots, \mathfrak{g}_{n}$ be simple Lie algebras. Denote by $d_{i}$ the minimal dimension of a nontrivial representation of $\mathfrak{g}_{i}$. Let $\psi$ be a representation of the semisimple algebra $\oplus_{i} \mathfrak{g}_{i}$. If $\operatorname{dim} \psi<\sum_{i} d_{i}$ then $\psi$ is trivial on some $\mathfrak{g}_{i}$.

PROOF. Each irreducible component of $\psi$ is of the form

$$
\psi_{i_{1}} \otimes \psi_{i_{2}} \otimes \cdots \otimes \psi_{i_{k}}
$$

where $\psi_{i_{j}}$ is a nontrivial irreducible representation of $\mathfrak{g}_{i_{j}}$. The dimension of (7) is not less than

$$
d_{i_{1}} d_{i_{2}} \ldots d_{i_{k}} \geq d_{i_{1}}+d_{i_{2}}+\cdots+d_{i_{k}} .
$$

Therefore, if $\psi$ is nontrivial on each $\mathfrak{g}_{i}$ then $\operatorname{dim} \psi \geq \sum_{i} d_{i}$.

Let $\mathrm{A} \subset \Pi \backslash \Sigma$ and $\mathrm{B} \subset \Sigma$. In the sequel we consider representations $\varphi$ of $P$ with highest weights $\Lambda$ satisfying

$$
(\Lambda, \alpha)<0,(\Lambda, \beta)=0 \text { for all } \alpha \in \mathrm{A}, \beta \in \mathrm{B} .
$$

We define a class of positive roots $\delta$ of $\mathfrak{g}$ (significant (A, B)-roots) such that if $\Lambda+\gamma$ is regular then $(\Lambda+\gamma, \delta)<0$. By Bott's theorem and Lemma 1, this implies that $H^{q}\left(M, \mathcal{E}_{\varphi}\right)=0$ for $0 \leq q<\ell(\mathrm{A}, \mathrm{B})$, where $\ell(\mathrm{A}, \mathrm{B})$ is the number of significant (A, B)-roots.

Let us introduce the required concepts. A positive root

$$
\delta=\sum_{\alpha \in \mathrm{A}} c_{\alpha}(\delta) \cdot \alpha+\sum_{\beta \in \mathrm{B}} c_{\beta}(\delta) \cdot \beta, c_{\alpha}(\delta), c_{\beta}(\delta) \geq 0,
$$

of $\mathfrak{g}$ with

$$
c_{\alpha_{0}}(\delta)>0 \text { for some } \alpha_{0} \in \mathrm{A}
$$

will be called an (A,B)-root. Denote by $\Delta_{\mathrm{A}, \mathrm{B}}$ the set of (A, B)-roots. We say that $\delta \in \Delta_{\mathrm{A}, \mathrm{B}}$ is significant if there exists a root $\sigma_{\delta} \in \Delta_{\mathrm{A}, \mathrm{B}}$ such that

$$
\sigma_{\delta} \leq \delta, \quad \sum_{\alpha \in \mathrm{A}} c_{\alpha}\left(\sigma_{\delta}\right) \cdot(\alpha, \alpha) \geq \sum_{\beta \in \mathrm{B}} c_{\beta}\left(\sigma_{\delta}\right) \cdot(\beta, \beta),
$$

and $\mathrm{C}\left(\delta-\sigma_{\delta}\right)$ consists of simple roots of the same length not greater than $\left|\sigma_{\delta}\right|$. 
LEMMA 4. If a weight $\Lambda$ satisfies (8) and $\Lambda+\gamma$ is regular then for each significant $\delta \in \Delta_{\mathrm{A}, \mathrm{B}}$ one has $(\Lambda+\gamma, \delta)<0$.

Proof. By (8) and (3), we have

$$
\left(\Lambda+\gamma, \sigma_{\delta}\right)=\sum_{\alpha \in \mathrm{A}}\left(c_{\alpha}\left(\sigma_{\delta}\right) \cdot(\Lambda, \alpha)+\frac{1}{2} c_{\alpha}\left(\sigma_{\delta}\right) \cdot(\alpha, \alpha)\right)+\sum_{\beta \in \mathrm{B}} \frac{1}{2} c_{\beta}\left(\sigma_{\delta}\right) \cdot(\beta, \beta)
$$

Note that

$$
\frac{2(\Lambda, \alpha)}{(\alpha, \alpha)} \neq-1 \text { for each } \alpha \in \Pi
$$

because otherwise $(\Lambda+\gamma)(\alpha)=0$ and $\Lambda+\gamma$ is singular. Combining (2), (8), and (12), we obtain

$$
\frac{2(\Lambda, \alpha)}{(\alpha, \alpha)} \leq-2 \text { for each } \alpha \in \mathrm{A} .
$$

From (11), 13), and (10) one gets

$$
\left(\Lambda+\gamma, \sigma_{\delta}\right) \leq \sum_{\alpha \in \mathrm{A}}-\frac{1}{2} c_{\alpha}\left(\sigma_{\delta}\right) \cdot(\alpha, \alpha)+\sum_{\beta \in \mathrm{B}} \frac{1}{2} c_{\beta}\left(\sigma_{\delta}\right) \cdot(\beta, \beta) \leq 0 .
$$

By Lemma 2, there exist $\delta_{0}, \delta_{1}, \ldots, \delta_{m} \in \Delta_{\mathrm{A}, \mathrm{B}}$ such that $\delta_{0}=\sigma_{\delta}, \delta_{m}=\delta$, and

$$
\delta_{i}-\delta_{i-1} \in \mathrm{C}\left(\delta-\sigma_{\delta}\right) \subset \mathrm{A} \cup \mathrm{B} .
$$

Denote

$$
c=\frac{1}{2}\left(\delta_{i}-\delta_{i-1}, \delta_{i}-\delta_{i-1}\right) .
$$

By the definition of significant roots, $c$ does not depend on $i=1, \ldots, m$ and

$$
2 c \leq\left(\sigma_{\delta}, \sigma_{\delta}\right) .
$$

For any roots $\alpha, \beta$ if $|\alpha| \geq|\beta|$ then $\frac{(\alpha, \alpha)}{(\beta, \beta)} \in \mathbb{N}$. Applying this to $\delta_{0}=\sigma_{\delta}$ and $\delta_{i}-\delta_{i-1}$, from (16) we get $\frac{1}{2 c}\left(\delta_{0}, \delta_{0}\right) \in \mathbb{N}$ and, therefore, from (2) one obtains

$$
\frac{1}{c}\left(\Lambda+\gamma, \delta_{0}\right)=\frac{2\left(\Lambda+\gamma, \delta_{0}\right)}{\left(\delta_{0}, \delta_{0}\right)} \cdot \frac{\left(\delta_{0}, \delta_{0}\right)}{2 c} \in \mathbb{Z}
$$

By (2) and (3),

$$
\frac{1}{c}\left(\Lambda+\gamma, \delta_{i}-\delta_{i-1}\right)=\frac{1}{c}\left(\Lambda, \delta_{i}-\delta_{i-1}\right)+1 \in \mathbb{Z} .
$$

Moreover, from (15) and (8) we have $\frac{1}{c}\left(\Lambda, \delta_{i}-\delta_{i-1}\right) \leq 0$, hence

$$
\frac{1}{c}\left(\Lambda+\gamma, \delta_{i}-\delta_{i-1}\right) \leq 1 .
$$

By induction on $i$, combining (14), (17), (18), and (19), we get $\frac{1}{c}\left(\Lambda+\gamma, \delta_{i}\right) \in \mathbb{Z}$ and

$$
\left(\Lambda+\gamma, \delta_{i}\right)<0 \text { for all } i=0,1, \ldots, m
$$

because otherwise $\left(\Lambda+\gamma, \delta_{i}\right)=0$ for some positive root $\delta_{i}$ and $\Lambda+\gamma$ is singular. In particular, $(\Lambda+\gamma, \delta)<0$.

EXAMPLE 1. We prove three sufficient conditions for $\delta \in \Delta_{\mathrm{A}, \mathrm{B}}$ to be significant by giving corresponding $\sigma_{\delta}$. 
(1) If $\mathrm{C}(\delta)$ contains simple roots of the same length, we can, by (9), take $\sigma_{\delta} \in \mathrm{C}(\delta) \cap \mathrm{A}$.

(2) If there is $\alpha_{0} \in \mathrm{C}(\delta) \cap \mathrm{A}$ longer than the other roots from $\mathrm{C}(\delta) \backslash\left\{\alpha_{0}\right\}$, then $\mathrm{C}(\delta) \backslash\left\{\alpha_{0}\right\}$ contains roots of the same length, $c_{\alpha_{0}}(\delta)=1$, hence $\mathrm{C}\left(\delta-\alpha_{0}\right)=\mathrm{C}(\delta) \backslash\left\{\alpha_{0}\right\}$ and one can put $\sigma_{\delta}=\alpha_{0}$.

(3) If there exists $\alpha_{0} \in \mathrm{C}(\delta) \cap \mathrm{A}$ shorter than the other roots from $\mathrm{C}(\delta) \backslash\left\{\alpha_{0}\right\}$ and $c_{\alpha_{0}}(\delta)=-\frac{2\left(\beta, \alpha_{0}\right)}{\left(\alpha_{0}, \alpha_{0}\right)}$ for some $\beta \in \mathrm{C}(\delta) \backslash\left\{\alpha_{0}\right\}$, then $\mathrm{C}(\delta) \backslash\left\{\alpha_{0}\right\}$ consists of roots of length $|\beta|=\left|\beta-\frac{2\left(\beta, \alpha_{0}\right)}{\left(\alpha_{0}, \alpha_{0}\right)} \cdot \alpha_{0}\right|$ and we set

$$
\sigma_{\delta}=\beta-\frac{2\left(\beta, \alpha_{0}\right)}{\left(\alpha_{0}, \alpha_{0}\right)} \cdot \alpha_{0} .
$$

Let $\ell(A, B)$ be the number of significant $(A, B)$-roots. This number can be computed in terms of some graphs, if we regard A and B as subgraphs of the Dynkin diagram D of $\mathfrak{g}$.

ExAmple 2. Denote by $\mathrm{U}$ the union of the connected components $\mathrm{C}$ of $\mathrm{A} \cup \mathrm{B}$ satisfying $\mathrm{C} \cap \mathrm{A}=\emptyset$ and set $\mathrm{B}^{\prime}=\mathrm{B} \backslash \mathrm{U}$. Clearly, (A, B)-roots coincide with $\left(\mathrm{A}, \mathrm{B}^{\prime}\right)$-roots and $\ell(\mathrm{A}, \mathrm{B})=\ell\left(\mathrm{A}, \mathrm{B}^{\prime}\right)$ because for each $(\mathrm{A}, \mathrm{B})$-root $\delta$ the subgraph $\mathrm{C}(\delta) \subset \mathrm{A} \cup \mathrm{B}$ is connected and, by (9), $\mathrm{C}(\delta) \cap \mathrm{A} \neq \emptyset$.

Suppose that all the edges of $\mathrm{A} \cup \mathrm{B}^{\prime}$ are simple, that is, each connected component of $\mathrm{A} \cup \mathrm{B}^{\prime}$ contains simple roots of the same length. Then, by Example $\mathbb{1}$, each $\left(A, B^{\prime}\right)$-root is significant, hence $\ell(A, B)$ equals the number of $\left(A, B^{\prime}\right)$-roots. The latter is equal to the number of positive roots of $\mathfrak{g}_{A \cup B^{\prime}}$ minus the number of positive roots of $\mathfrak{g}_{\mathrm{B}^{\prime}}$, where $\mathfrak{g}_{\mathrm{A} \cup \mathrm{B}^{\prime}}$ and $\mathfrak{g}_{\mathrm{B}^{\prime}}$ are semisimple Lie algebras with Dynkin diagrams $\mathrm{A} \cup \mathrm{B}^{\prime}$ and $\mathrm{B}^{\prime}$ respectively.

In what follows, we will denote by the same letter a representation $\varphi: P \rightarrow$ $\mathrm{GL}(E)$ and its differential $\varphi: \mathfrak{p} \rightarrow \mathfrak{g l}(E)$.

THEOREM 1. Consider an irreducible representation $\varphi$ of $P$ with highest weight $\Lambda \in \mathfrak{t}^{*}$. Let $\mathrm{A}$ and $\mathrm{B}$ be subsets of $\Pi \backslash \Sigma$ and $\Sigma$ respectively such that (\$) holds. Then we have $H^{q}\left(M, \mathcal{E}_{\varphi}\right)=0$ for $0 \leq q<\ell(\mathrm{A}, \mathrm{B})$.

Proof. If $\Lambda+\gamma$ is singular then, by Bott's theorem, $H^{*}\left(M, \mathcal{E}_{\varphi}\right)=0$; suppose that $\Lambda+\gamma$ is regular. By Lemma 4 , $(\Lambda+\gamma, \delta)<0$ for each significant $\delta \in \Delta_{\mathrm{A}, \mathrm{B}}$. Therefore, the index of $\Lambda+\gamma$ is not less than $\ell(\mathrm{A}, \mathrm{B})$. Applying Bott's theorem, one completes the proof.

We say that a representation $\varphi: P \rightarrow \mathrm{GL}(E)$ is obtained by natural operations from a representation $\tilde{\varphi}: P \rightarrow \operatorname{GL}(\tilde{E})$ if there is a homomorphism $\pi: \operatorname{GL}(\tilde{E}) \rightarrow$ $\mathrm{GL}(E)$ such that

$$
\varphi=\pi \circ \tilde{\varphi}
$$

ExAmPLE 3. A representation $\varphi$ obtained from $\tilde{\varphi}$ by tensor operations (11), for instance $\varphi=\tilde{\varphi} \tilde{\varphi}^{*}$, satisfies 21 .

Denote by $H^{\prime}$ and $\mathfrak{h}^{\prime}$ the semisimple commutator subgroup of $H$ and its tangent subalgebra respectively. Let us regard $\Sigma$ as a subdiagram of the Dynkin diagram D of $\mathfrak{g}$. Then $\mathfrak{h}^{\prime}$ is a semisimple Lie algebra with Dynkin diagram $\Sigma$. As usually, each connected component $\mathrm{C}$ of $\Sigma$ determines a simple ideal $\mathfrak{h}_{\mathrm{C}}$ of $\mathfrak{h}^{\prime}$. If a representation $\tilde{\varphi}$ of $P$ is trivial on some $\mathfrak{h}_{\mathrm{C}}$ then, by (21), any $\varphi$ obtained from $\tilde{\varphi}$ by natural 
operations is also trivial on $\mathfrak{h}_{\mathrm{C}}$. This observation, Lemmas 1. 3, and Theorem 1 allow to prove that if $\varphi$ is obtained by natural operations from a representation of relatively small dimension then some cohomology groups $H^{q}\left(M, \mathcal{E}_{\varphi}\right)$ are zero.

Indeed, Lemma 3 guarantees that $\tilde{\varphi}$ and, therefore, $\varphi$ are trivial on some ideals of $\mathfrak{h}^{\prime}$ if the dimension of $\tilde{\varphi}$ is sufficiently small. The less is the dimension of $\tilde{\varphi}$ the more ideals $\varphi$ is trivial on. By the construction (伍) of the corresponding completely reducible representation $\varphi^{s}, \varphi\left(\mathfrak{h}_{\mathrm{C}}\right)=0$ implies $\varphi^{s}\left(\mathfrak{h}_{\mathrm{C}}\right)=0$. Let B be the union of those connected components $\mathrm{C}$ of $\Sigma$ for which one has $\varphi^{s}\left(\mathfrak{h}_{\mathrm{C}}\right)=0$.

Consider an arbitrary irreducible component $\varphi^{\prime}$ of $\varphi^{s}$ with highest weight $\Lambda$. Clearly, $(\Lambda, \beta)=0$ for each $\beta \in \mathrm{B}$. We set

$$
\mathrm{A}\left(\varphi^{\prime}\right)=\{\alpha \in \Pi \backslash \Sigma:(\Lambda, \alpha)<0\} .
$$

If $\mathrm{A}\left(\varphi^{\prime}\right)$ is empty then $\Lambda$ is dominant and, by Bott's theorem, $H^{q}\left(M, \mathcal{E}_{\varphi^{\prime}}\right)=0$ for all $q>0$. Otherwise, according to Theorem 1 1 , we get $H^{q}\left(M, \mathcal{E}_{\varphi^{\prime}}\right)=0$ for $0 \leq q<\ell\left(\mathrm{A}\left(\varphi^{\prime}\right), \mathrm{B}\right)$. Thus, by Lemma $\mathrm{f}$.

$$
H^{q}\left(M, \mathcal{E}_{\varphi^{s}}\right)=H^{q}\left(M, \mathcal{E}_{\varphi}\right)=0 \text { for all } 0<q<\min _{\varphi^{\prime}} \ell\left(\mathrm{A}\left(\varphi^{\prime}\right), \mathrm{B}\right),
$$

where $\varphi^{\prime}$ runs through the irreducible components of $\varphi^{s}$ with $\mathrm{A}\left(\varphi^{\prime}\right) \neq \emptyset$.

The following theorem is an example of such a result. To formulate it we need some notations. We say that a subset $\mathrm{B} \subset \Sigma$ is adjacent to a vertex $\alpha \in \Pi \backslash \Sigma$ of $\mathrm{D}$ if there is an edge of $\mathrm{D}$ connecting $\alpha$ and $\mathrm{B}$. Equivalently, there is a unique simple root $\beta \in \mathrm{B}$ such that $(\alpha, \beta) \neq 0$. For a simple root $\alpha \in \Pi \backslash \Sigma$ consider the connected components $\mathrm{C}_{1}, \ldots, \mathrm{C}_{n}$ of $\Sigma \subset \mathrm{D}$ adjacent to $\alpha$ and the corresponding simple ideals $\mathfrak{h}_{\mathrm{C}_{1}}, \ldots, \mathfrak{h}_{\mathrm{C}_{n}}$ of $\mathfrak{h}^{\prime}$. Denote by $d_{i}$ the minimal dimension of a nontrivial representation of $\mathfrak{h}_{C_{i}}$ and set

$$
\begin{aligned}
& d(\alpha)=d_{1}+\cdots+d_{n}, \\
& \ell(\alpha)=\min _{i=1, \ldots, n} \ell\left(\{\alpha\}, \mathrm{C}_{i}\right) .
\end{aligned}
$$

If there are no nonempty connected components adjacent to $\alpha$, we put $d(\alpha)=$ $\ell(\alpha)=1$. Set also

$$
\begin{aligned}
& d(P)=\min _{\alpha \in \Pi \backslash \Sigma} d(\alpha), \\
& \ell(P)=\min _{\alpha \in \Pi \backslash \Sigma} \ell(\alpha) .
\end{aligned}
$$

THEOREM 2. Suppose that a representation $\varphi$ of $P$ is obtained by natural operations from a representation $\tilde{\varphi}$ of dimension less then $d(P)$. Then we have

$$
H^{q}\left(M, \mathcal{E}_{\varphi}\right)=0 \text { for } 0<q<\ell(P) \text {. }
$$

Proof. If $d(P)=1$, the statement is trivial. If $d(P)>1$ then from Lemma 3 it follows that for each $\alpha \in \Pi \backslash \Sigma$ there is a nonempty connected component $\mathrm{C}_{\alpha}$ of $\Sigma$ adjacent to $\alpha$ such that $\tilde{\varphi}$ and, by (21), $\varphi$ are trivial on $\mathfrak{h}_{\mathrm{C}_{\alpha}}$. By the definition of $\ell(P)$, we have $\ell(P) \leq \ell\left(\{\alpha\}, \mathrm{C}_{\alpha}\right)$ and from (22) one obtains (23).

Remark 1. We have $d(P)>1$ if and only if for each $\alpha \in \Pi \backslash \Sigma$ there exists a nonempty connected component $\mathrm{C} \subset \Sigma$ adjacent to $\alpha$. According to the following lemma, this is also equivalent to $\ell(P)>1$. In this case the result of Theorem 2 is nontrivial. 
Lemma 5. If $\mathrm{B}$ is a nonempty subset of $\Sigma$ adjacent to $\alpha \in \Pi \backslash \Sigma$ then

$$
\ell(\{\alpha\}, \mathrm{B}) \geq 2 .
$$

Proof. The root $\alpha$ is clearly a significant $(\alpha, \mathrm{B})$-root. By assumption, there is $\beta \in \mathrm{B}$ such that $(\alpha, \beta) \neq 0$. Consider the root $\delta$ of the form

$$
\delta=a \cdot \alpha+b \cdot \beta, \quad a, b>0,
$$

such that $\delta+\alpha$ and $\delta+\beta$ are not roots of $\mathfrak{g}$. According to Example $\mathbb{1}$, $\delta$ is also significant. Thus we have at least two distinct significant roots.

Example 4. Consider the Grassmanian $\mathrm{Gr}_{n, k}$ of $k$-dimensional subspaces of $\mathbb{C}^{n}$. It is a flag manifold of the group $\mathrm{SL}_{n}(\mathbb{C})$. The stabilizer of a point $o \in \mathrm{Gr}_{n, k}$ is a parabolic subgroup with $\Sigma=\Pi \backslash\{\alpha\}$, where $\alpha$ is the $k$-th simple root in the Dynkin diagram

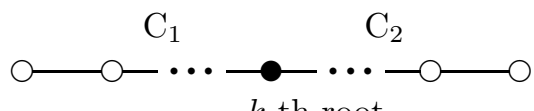

$k$-th root

of $\mathrm{SL}_{n}(\mathbb{C})$. The subdiagram $\Sigma$ consists of two components $\mathrm{C}_{1}$ and $\mathrm{C}_{2}$ adjacent to $\alpha$, one of which is empty if $\mathrm{Gr}_{n, k} \simeq \mathbb{C P}^{n-1}$, that is, if $k=1$ or $k=n-1$. Hence $d(P)=n$ if $1<k<n-1$, and $d(P)=n-1$ if $k=1$ or $k=n-1$. According to Example 2, we have $\ell\left(\{\alpha\}, \mathrm{C}_{1}\right)=k, \ell\left(\{\alpha\}, \mathrm{C}_{2}\right)=n-k$. Thus $\ell(P)=\min \{k, n-k\}$ if $1<k<n-1$, and $\ell(P)=n-1$ if $k=1$ or $k=n-1$. This computation gives the following result.

TheOREM 3. For any vector bundle $\mathbf{E} \rightarrow \mathrm{Gr}_{n, k}, 1<k<n-1$, obtained by tensor operations from a homogeneous vector bundle $\tilde{\mathbf{E}} \rightarrow \mathrm{Gr}_{n, k}$ of rank less than $n$ one has $H^{q}\left(\mathrm{Gr}_{n, k}, \mathcal{E}\right)=0$ for all $0<q<\min \{k, n-k\}$.

Proof. It is known (see [6]) that for $1<k<n-1$ any transitive Lie subgroup

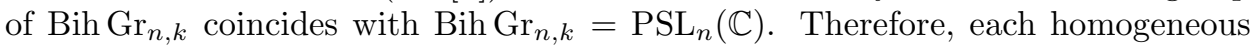
vector bundle over $\mathrm{Gr}_{n, k}$ is homogeneous with respect to the simply connected group $\mathrm{SL}_{n}(\mathbb{C})$. Hence $\tilde{\mathbf{E}}=\mathbf{E}_{\tilde{\varphi}}$ for some representation $\tilde{\varphi}$ of $P \subset \mathrm{SL}_{n}(\mathbb{C})$. By (1) , we have $\mathbf{E}=\mathbf{E}_{\varphi}$, where $\varphi$ is obtained from $\tilde{\varphi}$ by tensor operations. According to Example 3, Theorem 2, and the above computation of $d(P)$ and $\ell(P)$, we complete the proof.

Cohomology groups of sheaves considered here appear, in particular, in deformation theory [8]. Theorems 1 and 2 allow to prove rigidity, that is, absence of nontrivial deformations [8], of some homogeneous vector bundles and supermanifolds [5, 7]. A result on rigidity of vector bundles is given by the following theorem, while homogeneous supermanifolds are studied in [4].

TheOREM 4. For any representation $\varphi$ of $P$ obtained by natural operations from a representation $\tilde{\varphi}$ of dimension less than $d(P)$ the bundle $\mathbf{E}_{\varphi}$ is rigid.

Proof. By (1), we have $\mathbf{E}_{\varphi} \otimes \mathbf{E}_{\varphi}^{*}=\mathbf{E}_{\varphi \varphi^{*}}$. According to Example 3, the representation $\varphi \varphi^{*}$ is also obtained from $\tilde{\varphi}$ by natural operations. Then from Theorem 2 and Remark 1 one gets $H^{1}\left(M, \mathcal{E}_{\varphi} \otimes \mathcal{E}_{\varphi}^{*}\right)=0$, which implies that $\mathbf{E}_{\varphi}$ is rigid (see [3, 8]). 


\section{Acknowledgements}

I would like to express deep gratitude to Prof. A. L. Onishchik for his attention to this work and many useful remarks. It is a pleasure to thank the organizers of the European Summer School 2001 on Asymptotic Combinatorics with Application to Mathematical Physics in St. Petersburg for the invitation and providing a stimulating environment. I am also grateful to the Erwin Schrödinger Institute in Vienna for hospitality in October-November 2000 where a part of this research was done.

\section{References}

[1] D.N. Akhiezer. Lie group actions in complex analysis. Vieweg \& Sohn, Braunschweig, 1995.

[2] R. Bott. Homogeneous vector bundles. Ann. of Math. 66 (1957) no. 2, 203-248.

[3] O. Forster, K. Knorr. Über die Deformationen von Vektorraumbündeln auf kompakten komplexen Räumen. Math. Ann. 209 (1974) no. 4, 291-346.

[4] S. Igonin. Homogeneous supermanifolds associated with Hermitian symmetric spaces. In preparation.

[5] Yu.I. Manin. Gauge field theory and complex geometry. Springer-Verlag, Berlin, 1988.

[6] A.L. Onishchik. Topology of transitive transformation groups. Barth Verlag, Leipzig, 1994.

[7] A.L. Onishchik. On the classification of complex analytic supermanifolds. Lobachevskii J. Math. 4 (1999), 47-70 (electronic).

[8] V.P. Palamodov. Deformations of complex spaces. Encycl. Math. Sci. 10 (1990), 105-194.

Independent University of Moscow; University of Twente, the Netherlands

E-mail address: igonin@mccme.ru 